Ältere Patienten

\title{
Mehr Pneumonien unter Neuroleptika
}

Trifiro G et al. Association of community-acquired pneumonia with antipsychotic

drug use in elderly patients. Ann Intern Med 2010;152:418-425

\section{Hintergrund und Fragestellung}

In Altersheimen erhalten bis zu 40\% der Bewohner Neuroleptika [1]. Im April 2005 veröffentlichte die amerikanische Arzneimittelbehörde (FDA) einen Warnhinweis zur Verschreibung von atypischen Neuroleptika wegen einer erhöhten Rate an kardio- und zerebrovaskulären Ereignissen sowie Infektionen unter der Therapie [2].

Eine niederländische Forschergruppe untersuchte in einer Kohortenstudie die Frage, ob ein Zusammenhang zwischen der Einnahme von Psychopharmaka und Pneumonien bei älteren Patienten (Alter $\geq 65$ Jahre) besteht.

\section{Methodik}

Von 1996 bis 2006 wurden mithilfe einer Datenbank (IPCI Data Base) Patienten identifiziert, die unter Neuroleptikatherapie an einer ambulant erworbenen Pneumonie (CAP) erkrankt waren. In der IPCI-Datenbank werden Patientendaten aus 300 Allgemeinarztpraxen in den Niederlanden erfasst. Die Kontrollgruppe umfasste bis zu 20 Personen pro Pneumoniefall $(\mathrm{n}=1686)$. Der Verbrauch an Neuroleptika wurde anhand der Datenbank untersucht. Mithilfe einer logistischen Regressionsanalyse wurde ein möglicher Zusammenhang mit dem Auftreten einer CAP überprüft.

\section{Ergebnisse}

258 Personen (Durchschnittsalter: 83 Jahre; Raucher: $14,3 \%$ ), die unter der Einnahme von Neuroleptika an einer CAP erkrankten, wurden in die Studie aufgenommen. 25,2\% der Betroffenen starben innerhalb von 30 Tagen an ihrer Erkrankung. Die drei häufigsten Begleiterkrankungen waren Diabetes mellitus $(22,5 \%)$, Herzinsuffizienz $(21,3 \%)$ und COPD (19,4\%).

Das Pneumonierisiko war bei der Einnahme atypischer bzw. herkömmlicher Neuroleptika um das 2,6-Fache (Odds Ratio [OR] $=2,61 ; 95 \%$-Konfidenzintervall [KI]: 1,48-4,81) bzw. um das 1,7-Fache (OR = 5,97; 95\%-KI: 1,49_ 23,98) erhöht.

Tabelle 1

Neuroleptika und Pneumonierisiko

Tödliche und nicht tödliche Pneumonien

Antipsychotische Medikamente Patienten Kontrollen

Adjustierte Odds Ratio (95\%-Konfidenzintervall)

\section{Derzeit angewendete Neuroleptika}

Atypische Neuroleptika
Herkömmliche Neuroleptika
Neuroleptika
Kürzlich angewendete Neuroleptika
Atypische Neuroleptika
Herkömmliche Neuroleptika
Atypische plus herkömmliche
Neuroleptika
$10,9 \%$ $40,7 \%$ $5,9 \%$ $2,61(1,48-4,61)$ $1,6 \%$ $30,6 \%$ $1,76(1,22-2,53)$ $0,5 \%$ $5,30(1,30-21,76)$ $1,9 \%$ $18,2 \%$ $2,3 \%$ $1.38(0,46-4,13)$ $0,4 \%$ $16,7 \%$ $1,31(0,84-2,04)$

$0,1 \%$

\section{Kommentar}

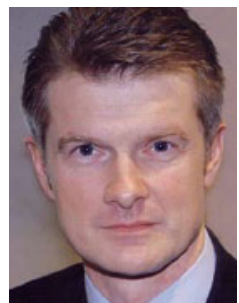

Prof. Dr. med.

Martin Kohlhäufl, Stuttgart-Gerlingen

Bereits im Jahr 1999 ergab eine Metaanalyse von 15 randomisierten Studien, dass bei älteren Demenzpatienten die Mortalität durch Neuroleptika um das 1,5-Fache gegenüber Placebo erhöht wird [3]. 2008 zeigte eine epidemiologische Registerstudie mit 2944 älteren Patienten (Durchschnittsalter: 81 Jahre), dass die Einnahme von Neuroleptika einen Anstieg des Pneumonierisikos um ca. $60 \%$ verursacht [4] Das Pneumonierisiko war in der ersten Behandlungswoche fast fünffach erhöht. Eine eindeutige Dosis-Wirkungs-Beziehung konnte jedoch im Gegensatz zu der nun vorliegenden aktuellen Kohortenstudie nicht belegt werden.

Als Ursachen für das unter Neuroleptika erhöhte Risiko, an einer CAP zu erkranken, werden eine erhöhte Aspirationsgefahr durch medikamentös induzierte pharyngeale Dyskinesien oder eine Behinderung des oropharyngealen Nahrungstransports durch medikamentös induzierte Mundtrockenheit angesehen. Ob die Neuroleptikatherapie bei bestimmten Risikopatienten nicht durchgeführt werden soll, kann die Studie nicht beantworten, da sie nicht randomisiert war.

\section{Fazit}

Bei Patienten mit einem erhöhten Pneumonierisiko (Asthma, COPD, Immunsuppression, alte Menschen) ist die Indikation zurTherapie mit Neuroleptika sorgfältig zu prüfen. Gerade bei Altersheimbewohnern werden Neuroleptika in mehr als der Hälfte der Fälle ohne eine entsprechende Indikation rezeptiert [5].

\footnotetext{
Literatur

1. Dewa CS et al. Clin Ther 2002;24:1466-1447

2. www.fda.gov/cder/drug/advisory/antipsychotics htm

3. Maixner SM et al. J Clin Pychiatry 1999;60 Supp 8:29-41

4. Knol W et al. J Am Geriatr Soc 2008;56:661-666

5. Lawlor BA.J Clin Psychiatry 2004;65, Suppl 11:5-10
} 